\title{
Targeted expression of a human pituitary tumor-derived isoform of FGF receptor-4 recapitulates pituitary tumorigenesis
}

\author{
Shereen Ezzat,, ${ }^{1,2}$ Lei Zheng, ${ }^{1,2}$ Xian-Feng Zhu, ${ }^{1,2}$ Gillian E. Wu, ${ }^{3,4}$ and Sylvia L. Asa ${ }^{2,4,5}$ \\ ${ }^{1}$ Department of Medicine, University of Toronto, Toronto, Ontario, Canada \\ ${ }^{2}$ The Freeman Centre for Endocrine Oncology, Mount Sinai Hospital, Toronto, Ontario, Canada \\ ${ }^{3}$ Department of Medical Biophysics, University of Toronto, Toronto, Ontario, Canada \\ ${ }^{4}$ Ontario Cancer Institute, University Health Network, Toronto, Ontario, Canada \\ ${ }^{5}$ Department of Pathology and Laboratory Medicine, University of Toronto, Toronto, Ontario, Canada \\ Address correspondence to: Sylvia L. Asa, Ontario Cancer Institute, 610 University Avenue, Room 4-302, Toronto, Ontario, \\ Canada M5G-2M9. Phone: (416) 946-2099; Fax: (416) 946-6579; E-mail: sylvia.asa@uhn.on.ca.
}

Received for publication August 22, 2001, and accepted in revised form October 29, 2001.

\begin{abstract}
It is estimated that up to one in five individuals develop pituitary gland tumors. Despite the common occurrence of these tumors, the pathogenetic mechanisms underlying their development remain largely unknown. We report the identification of a novel pituitary tumor-derived, $\mathrm{N}$-terminally truncated isoform of FGF receptor-4 (ptd-FGFR4). The corresponding mRNA results from alternative transcription initiation and encodes a polypeptide that lacks a signal peptide and the first two extracellular Ig-like domains. ptd-FGFR4 has a distinctive cytoplasmic residence, is constitutively phosphorylated, and is transforming in vitro and in vivo. Here we show that targeted expression of ptd-FGFR4, but not FGFR4, results in pituitary tumors that morphologically recapitulate the human disease.
\end{abstract}

J. Clin. Invest. 109:69-78 (2002). DOI:10.1172/JCI200214036.

\section{Introduction}

Pituitary disease results in diverse symptoms, ranging from sexual dysfunction and infertility to accelerated heart disease and cancer. The most common cause of abnormal pituitary function is a pituitary tumor, which can secrete excess pituitary hormones and/or interfere with normal production of regulatory hormones. These tumors occur in up to $20 \%$ of the population (1). Although they are common neoplasms, their cause remains unknown.

The pathogenesis of human neoplasia is the subject of extensive investigation. Some studies characterize alterations in human tumors and identify features that correlate with tumors but differ from normal tissue. Others have induced molecular alterations in mouse models, and identified resulting changes that they implicate in tumor pathogenesis. However, the former may be epiphenomena in human pathology, and the latter may have little relevance to the human situation. Pituitary tumorigenesis is an area where there is an abundance of correlative data concerning altered expression of, or responses to, hormonal and growth factor stimuli that remain unsubstantiated as causative of human disease (2).

Several mouse models have developed pituitary neoplasia, including those with deficient retinoblastoma $(\mathrm{Rb})$ gene product (3); however, human pituitary tumors do not have structural alterations of the $\mathrm{Rb}$ gene (2). Mice lacking the dopamine $\mathrm{D}_{2}$ receptor or the cyclin-dependent kinase inhibitor p27 display an agerelated pattern of pituitary hyperplasia that progresses to adenoma formation (4-8). However, these models are not reflective of the human situation, as patients with pituitary tumors do not exhibit the underlying hyperplasia identified in the animal model. Moreover, the vast majority of primary human pituitary tumors have intact dopamine $\mathrm{D}_{2}$ receptor and p27 genes (2). Transgenic mice overexpressing hypothalamic hormones such as growth hormone-releasing hormone develop pituitary adenomas after prolonged massive hyperplasia of the target cell type (9), but again, this model does not closely mimic the human situation, with the exception of the very rare patient with neoplastic overexpression of such factors (2). Although these tumors are an integral part of the multiple endocrine neoplasia type 1 (MEN-1) syndrome, examination of the $M E N-1$ tumor suppressor gene, an obvious candidate disease gene, revealed no loss of heterozygosity, no identifiable mutations, and no downregulation of activity in the far more common sporadic tumors $(10,11)$. Activating mutations of the $\mathrm{G}_{\mathrm{s} \alpha}$ protein have been identified in growth hormone-producing pituitary adenomas (12), but these mutations are implicated in only a minority of this subtype of pituitary tumor. The events leading to pituitary tumorigenesis in the vast majority of tumors, therefore, remain to be elucidated.

The identification of mechanisms of tumorigenesis should identify alterations in primary tumors and validate them as causative of disease. We report such an approach in the study of pituitary tumors. The pituitary is the site of synthesis and the target of several growth 
factors (13) that modulate hormone production and are believed to regulate, in part, pituitary cell growth. Several members of the FGF family have been implicated (14), including FGF-2 (also known as basic or bFGF), which was originally isolated from bovine pituitary (15) and is differentially expressed by pituitary tumor cells, with higher levels associated with the more aggressive tumors (16). FGF signaling is mediated through one of four FGF receptor (FGFR) genes that encode a complex family of transmembrane receptor tyrosine kinases (17). Our examination of FGFR1, FGFR2, and FGFR3 expression in pituitary tumors failed to identify gross alterations that could be implicated in tumorigenesis (18). Expression of FGFR4, on the other hand, did reveal differences that implicate this receptor in pituitary tumorigenesis. We therefore pursued characterization of FGFR4 expression in pituitary adenomas, and we now report a novel transforming, pituitary tumor-derived, N-terminally truncated FGFR4 isoform.

\section{Methods}

Characterization of human pituitary tissues. Pituitary tumors were obtained at surgery. Normal pituitary tissue was obtained at autopsy within 6 hours postmortem. Each tissue sample was divided, and one portion was fixed in formalin and embedded in paraffin for detailed histologic and immunohistochemical analysis. The second piece was snap-frozen in liquid nitrogen and stored at $-70^{\circ} \mathrm{C}$.

$R T-P C R$ analysis of $m R N A$. Total RNA was extracted by the guanidinium isothiocyanate method. One microgram of DNase-treated RNA was reverse-transcribed using $2.5 \mathrm{U} / \mathrm{ml}$ of murine leukemia virus reverse transcriptase, $2.5 \mathrm{mM} \mathrm{MgCl}_{2}, 1 \mathrm{mM}$ dNTP, $2.5 \mathrm{mM}$ random hexamers, and $1 \mathrm{U} / \mathrm{ml}$ RNase inhibitor. The integrity of RNA of each sample was assessed by amplification of the PGK-1 gene. The human breast cancer MCF-7 cell line, which expresses FGFR4 (18) was used as a positive control for FGFR4 analysis. PCR analyses of FGFR 4 mRNA were performed with the following sense primers: exon 1 ( $5^{\prime}$-TCGGGCTGTCTGCGGACCCTG- $3^{\prime}$ ), exon 2 codons 73-93 (5'-CCTGTTGGGGGTCCTGCTGAGTGTG-3'), exon 4 codons 478-490 (5'-CTTGCTGGGGGTACCTGTGCCTATT- $3^{\prime}$ ), exon 5 codons 539-558 ( $5^{\prime}$-TACCTGCGGGGAACACCGTC-3'), exon 6 codons 658-679 (5'CTGCGCCATCAGCACTGGAGT- $3^{\prime}$ ), exon 6 codons 726-744 (5'-GCCTGGTAGAGAACGCTGT-3'), and exon 6 codons $745-767$ (5'-GGCAGCATCCGCTATAACTACCTGC- $\left.3^{\prime}\right)$. The antisense primers used were exon 11 codons 1536-1557 (5'-CTGACCAAGCCAGCACTGTGGC-3'), and exon 18 codons 2441-2461 (5'TCGGGTCTGGGGTGCAGACA- $3^{\prime}$ ). The identity of all PCR products was verified by sequencing using an automated sequencer.

Northern blot analysis of $m R N A$. Twenty micrograms of DNase-treated RNA was electrophoresed on a $1 \%$ agarose gel, transferred to a nitrocellulose membrane, fixed with a UV crosslinker, prehybridized, and hybridized with a 500-bp fragment corresponding to the kinase region of the FGFR 4 cDNA. The blot was washed and then exposed for 2 weeks at $-70^{\circ} \mathrm{C}$. Equal loading of samples was verified by hybridization of the same blot for PGK-1.

Rapid amplification of cDNA ends. First-strand cDNA was generated by reverse transcription from total RNA in the presence of the sense SMART II (CLONTECH Laboratories Inc., Palo Alto, California, USA) oligonucleotide primer (5'-AAGCAGTGGTAACAACGCAGAGTACGCGGG$3^{\prime}$ ), followed by the addition of dCTPs to the $3^{\prime}$ end of the CDNAs, and PCR using the sense primer 5'-CTAATACGACTCACTATAGGGCAAGCAGTGGTAACAACGCAGAGT-3' and the antisense primer in exon 7 of FGFR $4\left(5^{\prime}\right.$-GGCTCCGAAGCTGCTGCCGTTGATGAC- $3^{\prime}$ ). PCR products were amplified with an abridged internal universal amplification primer (5'-AAGCAGTGGTAACAACGCAGAGT- $\left.3^{\prime}\right)$ and a nested antisense primer in exon 6 of FGFR4 $\left(5^{\prime}\right.$ CACAGCGTTCTCTACCAGGC- $3^{\prime}$ ) under the following conditions: $94^{\circ} \mathrm{C}$ for 20 seconds, $62^{\circ} \mathrm{C}$ for 30 seconds, and $72^{\circ} \mathrm{C}$ for 5 minutes. A similar strategy was used for $3^{\prime}$ rapid amplification of cDNA ends (RACE). PCR products generated were cloned into the PCR-II vector as directed by the manufacturer (Invitrogen Corp., Carlsbad, California, USA). Cloned inserts verified to contain FGFR4 by Southern blot analysis were sequenced using an automated sequencer.

Transfection and plasmid constructs. The HEK 293 cell line is devoid of endogenous FGFRs, and was used for transient transfection studies. NIH 3T3 cells were stably transfected for transformation assays. The entire coding region of human FGFR4 cDNA was obtained from the LTR2HXII vector (provided by J. Partanen, Mount Sinai Hospital, Toronto, Ontario, Canada), and subcloned into the pcDNA3.1 expression vector (Invitrogen Corp.). The ptd-FGFR4 isoform identified in primary pituitary tumors was subcloned into the TA vector, and subsequently into pcDNA3.1. Inserts were sequenced to confirm FGFR4 wild-type sequence and ptd-FGFR4 fidelity as compared with primary pituitary tumors. To identify the ATG translation start site responsible for the $65-\mathrm{kDa}$ protein, we independently mutated the two in-frame ATGs at nucleotides 614-616 and at 686-688 to CTG using a transformer site-directed mutagenesis kit (CLONTECH Laboratories Inc.), following the manufacturer's instructions. The mutation was confirmed by nucleotide sequencing.

Protein extraction and cell fractionation. Total protein was extracted from total cell lysates and quantified. Cell fractionation was performed by the hypotonic/NP-40 lysis method. Cells were washed in Tris-buffered saline, swollen in homogenization buffer consisting of $10 \mathrm{mM}$ Tris HCI ( $\mathrm{pH}$ 7.4), $10 \mathrm{mM} \mathrm{KCl}, 1.0 \mathrm{mM}$ EGTA, $5 \mathrm{mM}$ $\mathrm{Cl}_{2}, 1 \mathrm{mM}$ DTT, and $0.5 \mathrm{mM}$ PMSF, and vortexed in homogenization buffer containing $0.6 \%$ NP- 40 . Supernatant contained cytoplasm and plasma membranes. The pellet containing nuclear fraction was suspended in resuspension buffer composed of $250 \mathrm{mM}$ Tris ( $\mathrm{pH}$ 7.8), $60 \mathrm{mM} \mathrm{KCl}, 1 \mathrm{mM} \mathrm{DTT}$, and $1 \mathrm{mM}$ PMSF. 
Western blot analysis. Protein concentrations were determined using a protein assay from Bio-Rad Laboratories Inc. (Hercules, California, USA). Equal amounts of protein $(50 \mu \mathrm{g})$ from whole-cell lysates or from cytoplasmic or membrane fractions were solubilized in $2 \times$ SDS sample buffer, separated on $8 \%$ SDS-PAGE gels, and transferred to nitrocellulose membranes. To determine possible glycosylation, cell lysates were subjected to $\mathrm{N}$-glycosidase F treatment according to the manufacturer's protocol (Boehringer Mannheim, Indianapolis, Indiana, USA). Blots were incubated with an affinitypurified polyclonal rabbit antiserum directed against the C-terminus of human FGFR4 (Santa Cruz Biotechnology Inc., Santa Cruz, California, USA). Immunoprecipitation with anti-FGFR4 and immunoblotting with monoclonal anti-phosphotyrosine antibody 4G10 (1:400; UBI, Lake Placid, New York, USA) was performed to assess phosphotyrosine activity. A polyclonal antibody to rodent prolactin (PRL) (National Hormone and Peptide Program, National Institute of Diabetes and Digestive and Kidney Diseases, Bethesda, Maryland, USA) was used at a dilution of 1:4,000 for detection of circulating PRL.

Immunobistochemistry. Tissues were fixed in formalin and embedded in paraffin. Immunolocalization was detected with the streptavidin-biotin-peroxidase complex technique and visualized with 3,3'-diaminobenzidine. Pituitary hormones were detected using antibodies described previously $(4,5,18)$. For localization of FGFR4 and ptd-FGFR4, a polyclonal antiserum that recognizes the C-terminal tail of FGFR4 (Santa Cruz Biotechnology Inc.) was diluted 1:1,500. For subcellular localization, primary antiserum was localized with fluorescein-tagged secondary antibody and visualized with an MRC 600 confocal microscope from Bio-Rad Laboratories Inc. The specificity of all reactions was verified by replacing primary antibody with normal serum, by examining negative control tissues, and by preabsorbing primary antibody with purified peptide.

Cell metabolic labeling. Cells were incubated with DMEM lacking cysteine and methionine (ICN Pharmaceuticals Inc., Costa Mesa, California, USA) for 2 hours, followed by metabolic labeling with $100 \mu \mathrm{Ci} / \mathrm{ml}$ trans${ }^{35} \mathrm{~S}$-labeled methionine (ICN Pharmaceuticals Inc.) for 60 minutes. The cell culture was split into four fractions, and chasing medium containing $10 \times$ methionine and $10 \times$ cysteine was added to each fraction for an incubation period of 30 minutes, 2 hours, 4 hours, or 6 hours. Cells were washed with $2 \times$ PBS and prepared for gel analysis. Blots were scanned for densitometric analysis, and the slopes of the decay rates for each construct were compared in three independent experiments.

Mitogenic assay. Mitogenesis of NIH 3T3 cells stably transfected with FGFR4 or ptd-FGFR 4 cDNA to FGF-1 $(50 \mathrm{ng} / \mathrm{ml})$ was measured by $\left[{ }^{3} \mathrm{H}\right]$ thymidine incorporation. After confirming mRNA and protein expression by RT-PCR and Western blotting, stably transfected cells were grown in 6 -well plates $\left(5 \times 10^{4}\right.$ cells/well $)$ and preincubated for 48 hours in serum-free media with insulin $(5 \mu \mathrm{g} / \mathrm{ml})$ and transferrin $(5 \mu \mathrm{g} / \mathrm{ml})$. Cells were treated with or without FGF-1 $(50 \mathrm{ng} / \mathrm{ml}$; SigmaAldrich, St. Louis, Missouri) and $10 \mathrm{U} / \mathrm{ml}$ of heparin in serum-free medium for 24 hours at $37^{\circ} \mathrm{C}$. During the last 6 hours, cells were labeled with $1 \mu \mathrm{Ci} / \mathrm{ml}\left[{ }^{3} \mathrm{H}\right]$ thymidine, and the trichloroacetic acid-precipitable radioactivity associated with the cells was measured.

Transformation assays. To investigate anchorage-independent growth, NIH 3T3 cells $\left(1 \times 10^{4}\right)$ were plated in $0.3 \%$ agar containing DMEM with $10 \%$ FCS on top of a layer containing $0.5 \%$ agar, DMEM, and 10\% FCS. Colony assays were conducted with and without FGF as above. After 2 weeks, colonies of cell aggregates greater than $50 \mu \mathrm{m}$ in size were counted using an ocular grid and a phase-contrast inverted microscope (Leica, Wetzlar, Germany).

In vivo tumorigenesis. NIH 3 T3 cells stably transfected with FGFR4, ptd-FGFR4, or vector were injected subcutaneously into Swiss nude mice $\left(5 \times 10^{6}\right.$ cells per injection). Tumor development at the site of injection was evaluated twice a week. Grossly visible tumors were examined histologically to confirm fibroblast origin and FGFR4 expression, and to exclude incidental spontaneous neoplasms. The site of injection was examined histologically in all mice at the time of sacrifice (6 weeks).

Transgene construction. The ptd-FGFR4 and FGFR4 cDNAs were released from pcDNA3.1 using HindIII/XbaI, and then ligated into pGL-3 (Promega Corp., Madison, Wisconsin, USA), which provided an SV40 polyA signal (pGL-3-FGFR4). The rat PRL 3-kb promoter was excised from P3-Luc plasmid (provided by H. Elsholtz, University of Toronto, Toronto, Ontario, Canada) using BamH1/HindIII, and then ligated into the corresponding sites of psp73 (Promega Corp.). The PRL promoter was released using HindIII and KpnI. HindIII/KpnI sites were used to open pGL-3-FGFR4 for ligation of the PRL promoter at the $5^{\prime}$ end of FGFR4. This created the PRL-ptd-FGFR4 5.1-kb transgene and the PRL-FGFR4 5.8-kb transgene. These transgenes were purified from $1 \%$ agarose gels, treated with GENECLEAN II (Bio 101 Inc., Vista, California, USA) and microinjected into one-cell ICR mouse zygotes (5 ng) at the Samuel Lunenfeld Research Institute (SLRI; Toronto, Ontario, Canada).

Identification of transgenic mice. Two approaches were used to identify transgene carriers. Genomic tail DNA was digested with KpnI/SalI to release the entire transgene, followed by Southern blotting and hybridization with a digoxygenin-labeled probe corresponding to the PRL-FGFR4 transgene. For confirmation, DNA was subjected to PCR analysis using a sense primer in the rPRL promoter, and an antisense primer in exon 2 of FGFR4 for the full-length construct or in exon 6 of FGFR4 for the ptd-FGFR4 construct. The resulting 780-bp products for PRL-FGFR4 or 587-bp products for PRL-ptd-FGFR4 were electrophoresed on $1 \%$ agarose gels, followed by Southern blotting and hybridization. Plasmids containing the transgene served as positive controls for both analyses. 
a

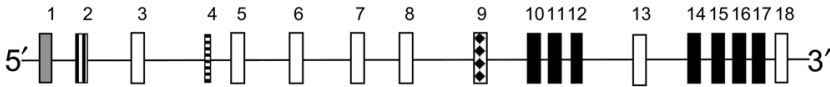

N

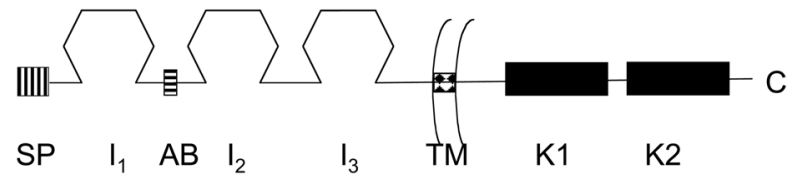

b

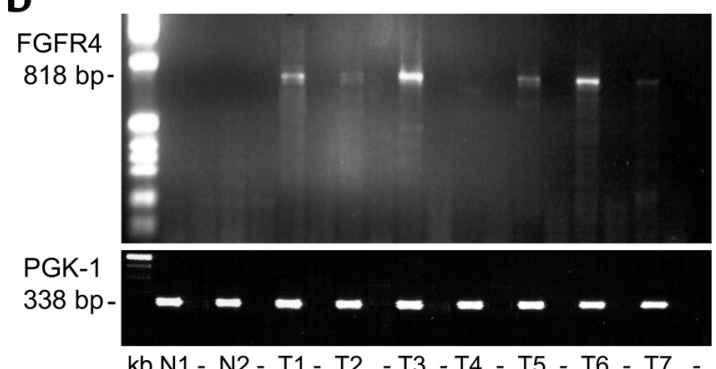

C

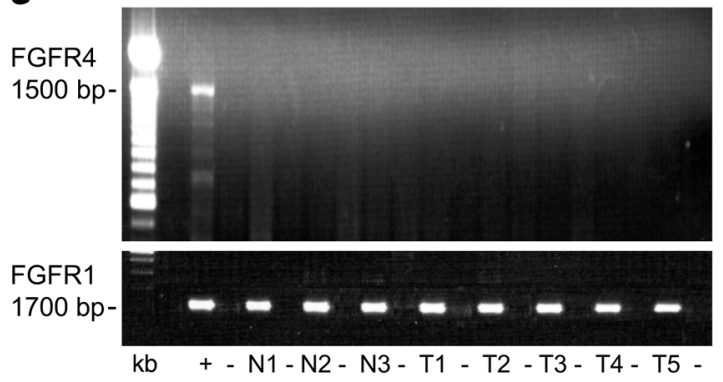

e

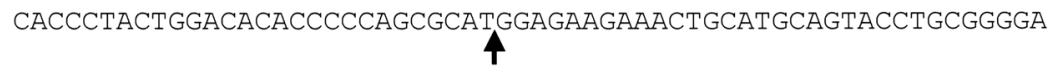
ACACCGTCAAGTTCCGCTGTCCAGCTGCAGGCAACCCCACGCCCACCATCCGCTGGCTTA AgGATGGACAGGCCTTTCATGGGGAGAACCGCATTGGAGGCATTCGGCTGGCGCCATCAG CACTGGAGTCTCGTGATGGAGAGCGTGGTGCCCTCGGACCGCGGCACATACACCTGCCTG GTAGAGAACGCTGTGGGCAGCATCCGCTATAACTACCTGCTAGATGTGCTGG
Figure 1

FGFR4 gene and protein structure, and RNA analysis. (a) The gene (top) contains 18 exons. The protein (bottom) is a transmembrane tyrosine kinase with three extracellular Ig-like loops. Exon 1 is untranslated. Exon 2 encodes signal peptide (SP). Exon 3 encodes first Ig-like domain $\left(I_{1}\right)$. Exon 4 encodes acid box $(A B)$. Exons 5 and 6 encode second Ig-like domain $\left(I_{2}\right)$. Exons 7 and 8 encode third Ig-like domain $\left(\mathrm{I}_{3}\right)$. Exon 9 encodes trans-membrane domain (TM). Exons 10-12 and 14-17 encode split kinase (K1 and K2). (b) RT-PCR of RNA from human pituitary tumors and normal pituitaries (N), identified an 818-bp product (exons 7-12) of FGFR4 in five of seven tumors, but not in normals. RNA integrity is indicated by RT-PCR for PGK-1 (bottom). Negative controls omitted reverse transcriptase (-). (c) Although the predicted 1,500-bp mRNA encoding full-length FGFR4 was present in MCF-7 breast cancer cells $(+)$, it is undetectable in normal pituitary and tumors. PCR on the same samples, using corresponding primers in signal peptide and kinase domains of FGFR1, resulted in products of the predicted 1,700-bp size (bottom). (d) Northern blot analysis of hFGFR4 expression. A $2.3 \mathrm{~kb}$ band is identified in RNA from MCF-7 cells $(+)$. Normal pituitary $(\mathrm{N})$ is negative; two pituitary tumors (T1 and T2) contain smaller bands of approximately $1.7 \mathrm{~kb}$. Hybridization with PGK-1 cDNA identifies a 1.8-kb species (bottom). (e) RACE. Arrow in the exon 5/6 sequence indicates the farthest $5^{\prime}$ transcription initiation site in $5^{\prime}$ RACE-generated products from pituitary tumors. The putative in-frame ATG translational start site is indicated in bold italics. (Sequence 492-782, following GenBank FGFR4 cDNA sequence accession no. X57205).
Animals. The care of animals was approved by the Institutional Animal Care facilities at SLRI. Mice were housed at the SLRI facility at $70-74^{\circ} \mathrm{C}$ and $50-75 \%$ humidity. Exposure to light for alternating 12-hour intervals was controlled automatically. Mice were fed an autoclaved formula diet and water ad libitum. At various ages, the animals were sacrificed with $\mathrm{CO}_{2}$ and underwent complete autopsy. Serum was collected for hormone analysis by Western blotting. Tissues were frozen in liquid nitrogen and stored at $-70^{\circ} \mathrm{C}$, or fixed in formalin and embedded in paraffin for histologic and immunohistochemical analysis. Frozen tissue (liver, heart, skeletal muscle, lung, and kidney) was used for mRNA expression analysis to confirm pituitary-specific expression of the transgene by Northern blotting.

Statistical analysis. Data are presented as mean \pm SE. Differences were assessed using the paired Student $t$ test. Significance level was assigned at $P<0.05$.

\section{Results}

The pituitary expresses a novel FGFR4 $m R N A$ species. In a survey of pituitary tumor gene expression, we identified expression of an FGFR4 species containing the third Iglike domain, transmembrane region, and kinase domain with C-terminal immunoreactivity (Figure 1a), in approximately $40 \%$ of pituitary tumors composed of the various hormone-secreting cell types, but not in nontumorous pituitary (18). We identified an mRNA transcript by RT-PCR using sense primers in exon 6 and antisense primers downstream of exon 10 (Figure 1b), but no PCR product was derived using sense primers upstream of exon 5 (Figure 1c). We excluded the possibility of mRNA splicing by examining the RNA using sense primers in exons 1,2, and 4; no products were identified (not shown). PCR performed on the same reverse-transcribed samples using corresponding primers in exons 2 through 11 of FGFR1 resulted in intact products of up to 1,700 bp in size (Figure 1c), confirming the intact nature of pituitary tumor RNA samples examined. Northern blotting and hybridization with a probe containing the kinase region of FGFR4 confirmed an mRNA species in pituitary tumors that was smaller than that encoding the fulllength receptor in control breast cancer cells (Figure 1d). 
To more precisely define the $5^{\prime}$ ends of this pituitary tumor-derived, N-terminally truncated FGFR4 cDNA, and to further exclude the possibility that alternative splicing or rearrangement was the mechanism responsible for this FGFR4 isoform, we adopted a $5^{\prime}$ RACE approach. We examined RNA from ten different primary human pituitary tumors. $5^{\prime}$ RACE products were cloned into TA vectors and screened by hybridization with full-length FGFR4 cDNA. At least ten clones from each pituitary tumor-derived cDNA sample were examined. There were no intronic sequences identified, excluding the possibility of genomic DNA contamination. Sequence analysis of these clones revealed transcription initiation in exon 5 , with the most $5^{\prime}$ nucleotide at position 520 of the human FGFR4 cDNA (Figure 1e). The 5' RACE-generated products contained two in-frame ATG translation initiation sites at nucleotides 614-616 and 686-688. All exon-intron boundaries conformed to the GT/AG rule. The existence of this $\mathrm{N}$-terminally truncated isoform in pituitary tumors was further verified by independently performed RT-PCR reactions using primers upstream of this site. Corresponding 3' RACE reactions from RNA of pituitary tumors yielded products with a common $3^{\prime}$ terminus containing no deletions or insertions.

Analysis of corresponding DNA from the same tumors failed to identify amplification, mutations, deletions, insertions, or rearrangements, making them unlikely mechanisms for the generation of this mRNA (not shown). Our findings are more consistent with the utilization of an alternative promoter situated in intron 4 of FGFR4 (19). We have named this pituitary tumor-derived variant ptd-FGFR4.

FGFR4 immunoreactivity in pituitary tumors is ptd-FGFR4. To investigate protein expression of FGFR4 and identify its cellular source in the pituitary, we examined multiple tumors and nontumorous pituitary by immunoblotting and immunohistochemistry, using an antibody that recognizes the C-terminus of FGFR4. Immunoblotting of FGFR4-immunoprecipitated protein from primary human pituitary tumors did not reveal a $110-\mathrm{kDa}$ immunoreactive protein indicative of full-length FGFR4. However, there was a product of approximately $65 \mathrm{kDa}$ that was found only in tumors, not in the normal pituitary (Figure 2a). By immunohistochemistry, nontumorous pituitary $(n=10)$ was consistently negative for FGFR4; $40 \%$ of pituitary tumors $(n=40)$ exhibited intense cytoplasmic staining for FGFR4 (Figure 2b).

To characterize the novel protein further, we expressed the mRNA identified in human pituitary tumors in cells that do not express endogenous FGFRs. Transfection of HEK 293 cells with full-length FGFR4 yields a product that migrates as a doublet of approximately $110 \mathrm{kDa}$ and $90 \mathrm{kDa}$ on Western blotting. Treatment with $\mathrm{N}$-glycosidase $\mathrm{F}$ results in a mobility decrease by both bands (not shown), consistent with the presence of $\mathrm{N}$-linked glycosylation in both, as recently reported (20). In contrast, transfection of the ptd-FGFR4 cDNA cloned from the primary human tumors yielded a product at approximately $65 \mathrm{kDa}$, the same size as that identified in Western blots of primary human pituitary tumors. Treatment with $\mathrm{N}$-glycosidase $\mathrm{F}$ did not alter the mobility of this $65-\mathrm{kDa}$ protein.

In view of the presence of two potential translation start sites, at nucleotides 614-616 and 686-688, we performed independent mutation of these sites. Mutation of the 686-688 ATG resulted in loss of FGFR4immunoreactive protein.

To assess the stability of the ptd-FGFR4 isoform, we performed pulse chase experiments using ${ }^{35} \mathrm{~S}$-methion-

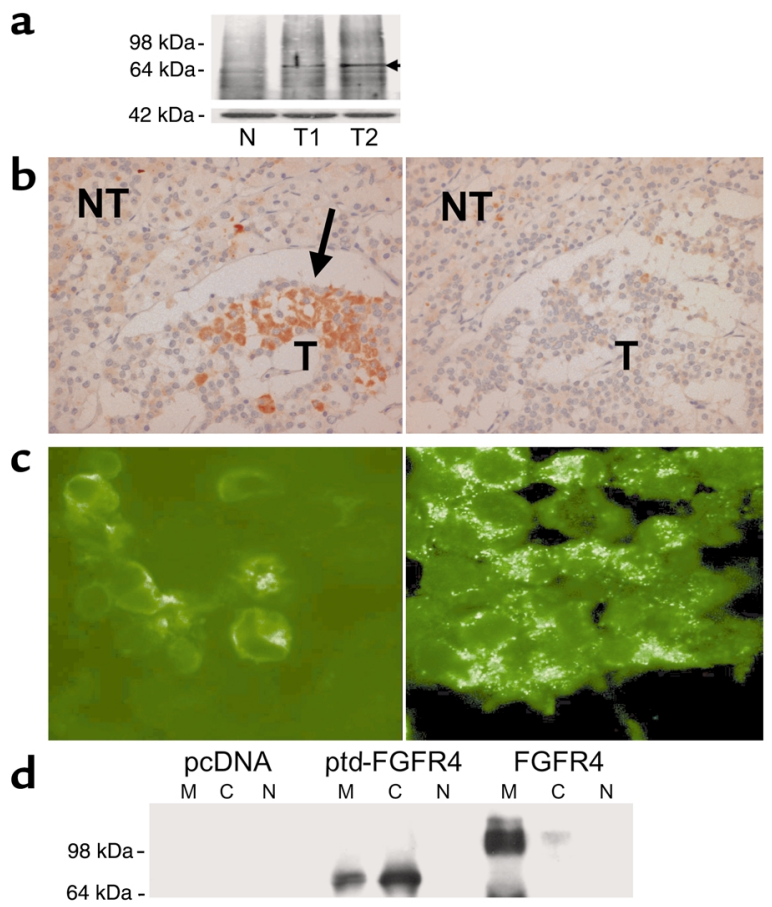

\section{Figure 2}

Characterization of ptd-FGFR4 protein. (a) Immunoprecipitation of normal and tumorous pituitary protein with anti-FGFR4, and immunoblotting with C-terminal anti-FGFR4, identifies no product at the size expected for full-length protein (approximately $110 \mathrm{kDa}$ ); instead there is a band that migrates at greater than $64 \mathrm{kDa}$ that is found in tumor samples (T1 and T2), but not in normal pituitary samples $(\mathrm{N})$. Equal amounts of protein that were immunoprecipitated and immunoblotted for actin are shown immediately below. (b) Immunohistochemical localization of FGFR4 reactivity in a human pituitary tumor. C-terminal FGFR4 immunoreactivity is seen in the cytoplasm of tumor ( $T$ ) cells (arrow), including those infiltrating around adjacent nontumorous (NT) pituitary cells that are immunonegative (left); absorption of primary antibody eliminates reactivity (right), proving the specificity of the reaction. (c) Using confocal microscopy, cells transfected with full-length FGFR4 (left) exhibit strong membrane-specific localization of immunoreactivity and irregular nuclear membranes, a phenomenon known as membrane ruffling. In contrast, cells transfected with ptd-FGFR4 (right) exhibit a diffuse cytoplasmic staining pattern. (d) Transfected full-length FGFR4 is detected as a $110-\mathrm{kDa}$ protein, predominantly in membrane fractions (M); transfected ptdFGFR4 migrates at $65 \mathrm{kDa}$ in cytoplasmic fractions (C) as well as in membrane fractions. Cells transfected with empty vector (pcDNA) are negative, and nuclear fractions $(\mathrm{N})$ are negative. 

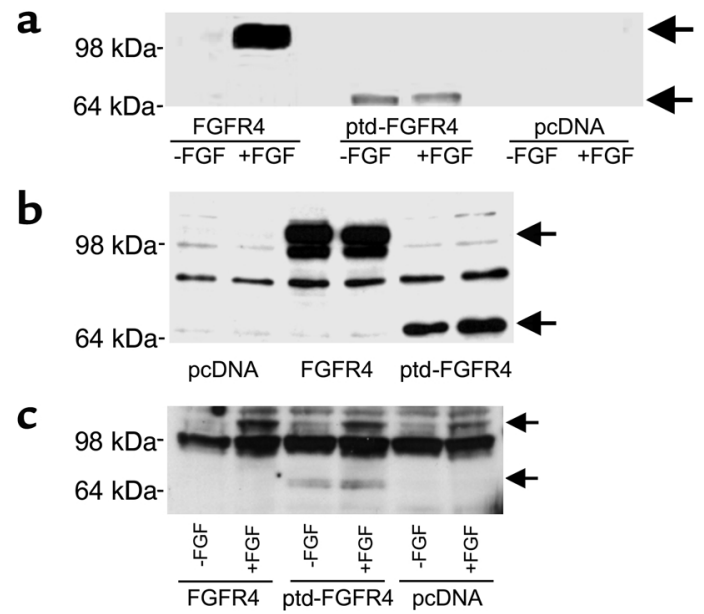

\begin{abstract}
Figure 3
Phosphotyrosine activity of FGFR4 and ptd-FGFR4. Transiently transfected HEK 293 cells (a) and stably transfected NIH 3 T3 cells (b and c) were exposed to FGF-1 $(50 \mathrm{ng} / \mathrm{ml}$; a and c). Equal amounts of protein were immunoprecipitated with an antibody directed against the C-terminus of FGFR4 and electrophoresed. (a) In HEK 293 cells, immunoblotting with anti-phosphotyrosine identifies a $110-\mathrm{kDa}$ fragment only after exposure to FGF-1 in cells transfected with full-length FGFR4 (upper arrow). Cells transfected with empty vector ( $p c D N A)$ are negative. Cells expressing ptd-FGFR4 exhibit phosphorylation of the $65-\mathrm{kDa}$ isoform with and without FGF-1 treatment, consistent with constitutive phosphorylation of the truncated receptor (lower arrow). (b) NIH 3 T3 cells stably transfected with FGFR4 express large amounts of the 110/90-kDa protein (upper arrow), and those stably transfected with ptd-FGFR4 express the 65-kDa protein (lower arrow); cells transfected with empty vector are negative. (c) NIH 3 T3 cells transfected with empty vector ( $p c D N A$, right) express endogenous FGFR4 that migrates at $110 \mathrm{kDa}$ and is detected with phosphotyrosine (PTYR) antibody after exposure to FGF-1 (upper arrow). Cells transfected with FGFR4 (left) exhibit the same pattern, but with greater intensity (documented by densitometry). Cells transfected with ptd-FGFR4 (middle) express a $65-\mathrm{kDa}$ protein that is phosphorylated in the presence and absence of FGF-1 (lower arrow); these cells also express the $110-k D a$ protein, and phosphorylation of this endogenous receptor is enhanced in the presence of FGF-1 to a greater degree than in control cells. These transfected NIH 3 T3 cells had FGFR4 immunoreactivity that comigrated with the PTYR-immunoreactive bands.
\end{abstract}

ine. During the course of a 6-hour chase, the $110 \mathrm{kDa}, 90$ $\mathrm{kDa}$, and $65 \mathrm{kDa}$ isoforms immunoprecipitated by the C-terminal antibody persisted, with similar decay rates as quantitated by densitometry. No $65-\mathrm{kDa}$ isoforms were identified in cells transfected with full-length FGFR4 (not shown), excluding degradation of the wildtype receptor as a propeptide of the $65-\mathrm{kDa}$ isoform.

ptd-FGR4 is cytoplasmic whereas FGFR4 is membraneanchored. To identify the subcellular localization of ptdFGFR4, we used confocal microscopy. HEK 293 cells transfected with full-length FGFR4 exhibited intense fluorescence at the cell periphery as previously described (21), indicating a membrane-anchored protein (Figure 2c, left). Cells expressing this receptor had a characteristic irregularity of the cell membrane, identified previously as "membrane ruffling" $(21,22)$, that was not seen in nontransfected cells (not shown). Cells transfected with ptd-FGFR4 displayed a markedly different pattern of staining, characteristic of a diffuse cytoplasmic protein (Figure 2c, right).

To corroborate these immunocytochemical findings, we performed Western blotting on subcellular fractions of transfected HEK 293 cells. Cells transfected with full-length FGFR4 showed predominant membrane reactivity for the 110/90-kDa doublet of FGFR4, minimal reactivity in cytoplasmic fractions, and no detectable reactivity in nuclear fractions. Cells transfected with ptd-FGFR4 showed cytoplasmic reactivity, some membrane reactivity, and undetectable nuclear reactivity for the $65-\mathrm{kDa}$ species (Figure $2 \mathrm{~d}$ ).

ptd-FGFR4 is constitutively phosphorylated. To determine the effect of FGFR4 N-terminal truncation on tyrosine phosphorylation, we analyzed HEK 293 cells transfected with FGFR4 or with ptd-FGFR4, under basal conditions and after exposure to FGF-1 for 9 hours (Figure 3a). We selected FGF-1 because this FGF ligand has been shown to bind all four FGFRs (23). Following immunoprecipitation with the antibody directed against the C-terminus of FGFR4, we identified the $110-\mathrm{kDa}$ fragment in cells transfected with intact FGFR4, and a 65-kDa fragment in cells transfected with ptd-FGFR4. Analysis of these FGFR4 immunoprecipitates using an anti-phosphotyrosine antibody revealed phosphorylation of the full-length receptor after exposure to FGF-1. In contrast, cells expressing ptd-FGFR4 exhibited phosphorylation of the $65-\mathrm{kDa}$ isoform with and without FGF-1 treatment, consistent with constitutive phosphorylation of the truncated receptor (Figure 3a).

To assess the proliferative potential of ptd-FGFR4 compared with wild-type FGFR4, we stably transfected NIH 3T3 cells (Figure 3b) and examined FGFR4 phosphorylation. The results were similar to those obtained in transiently transfected HEK 293 cells (Figure 3c). These cells also exhibited increased phosphorylation of the $110-\mathrm{kDa}$ fragment when transfected with ptd-FGFR4, suggesting that there may be an interaction between ptd-FGFR4 and endogenous full-length receptors.

ptd-FGFR4 is tumorigenic in vitro and in vivo. To determine whether the constitutive phosphorylation of ptdFGFR4 is of oncogenic significance, we used stably transfected NIH 3T3 cells expressing FGFR4 or ptdFGFR4 (Figure 3b). In keeping with previous reports suggesting that FGFR4 is not tumorigenic (24), $\left[{ }^{3} \mathrm{H}\right]$ thymidine incorporation by cells expressing FGFR4 was not significantly different from that of control cells transfected with empty vector, and addition of FGF-1 to culture media did not alter this finding. In contrast, cells expressing ptd-FGFR4 had a basal $\left[{ }^{3} \mathrm{H}\right]$ thymidine incorporation rate that was 1.7 times that of control cells, and incorporation was not further increased by exposure to FGF-1 in triplicate experiments.

Stably transfected NIH 3T3 cells expressing ptdFGFR4 developed anchorage independence, and after 
growing to confluence, they exhibited loss of contact inhibition (Figure 4a). The transforming effects of ptdFGFR 4 were therefore examined by assessing colony formation in soft agar. The efficiency of colony formation in soft agar, defined as percentage of innoculated cells that developed into colonies measuring $50 \mu \mathrm{m}$ or more (Figure $4 \mathrm{~b}$ ), was $0.22-0.30 \%$ by cells transfected with empty vector with or without FGF-1 stimulation. The efficiency of cells expressing FGFR4 under basal conditions was $0.30 \%$; addition of FGF- 1 increased this to $1.12 \%$. In contrast, cells expressing ptd-FGFR4 had a $5.04 \%$ efficiency of colony formation that increased to $7.56 \%$ when exposed to FGF-1.

Further evidence of tumorigenicity was the development of tumors in vivo. Injection of transfected NIH $3 \mathrm{~T} 3$ cells into nude mice resulted in large tumors when cells were transfected with ptd-FGFR4 ( $n=$ four of five mice), but not when transfected with FGFR4 ( $n=$ zero of five mice) or empty vector ( $n=$ zero of five mice) (Figure 4c). The lack of effect of transforming ability of the full-length wild-type FGFR4 is consistent with previous data (24), and was confirmed by histologic examination of the site of injection, which showed no viable injected fibroblasts.

Expression of ptd-FGFR4 causes pituitary tumors in transgenic mice. Convincing evidence of a direct role of ptdFGFR4 in pituitary tumorigenesis was obtained from transgenic mice. Using a PRL promoter that is expressed after completion of embryologic development (25), we generated transgenic mice selectively expressing ptd-FGFR4 in the pituitary. In addition to nontransgenic littermate controls, we generated transgenic mice expressing wild-type FGFR4 under the control of the same PRL promoter. Transgene carriers were identified by Southern blotting and by PCR, and two founders (each expressing one of the two transgenes) were propagated for further analysis. No extrapituitary transgene expression was identified. No pathology or developmental abnormalities were detected in the animals before 7 months of age, and the animals were fertile. At 7 months, the pituitaries of PRL-ptd-FGFR4 transgenic mice were of normal size; immunohistochemistry identified numerous lactotrophs with intense cytoplasmic FGFR4 expression. Diffuse hyperplasia like that previously noted in other models $(4,9)$ was not evident, since the reticulin stain did not show expansion of pituitary acini. The pituitaries of PRLFGFR4 transgenic mice contained lactotrophs with a similar intensity of FGFR4 immunoreactivity but with a distinct membrane pattern of staining.

By the age of 11 months, 90\% of PRL-ptd-FGFR 4 transgenic animals developed pituitary tumors (Figure 5, a and b). Circulating PRL levels of PRL-ptd-FGFR4 transgenic animals were higher than those of nontransgenic littermates, and correlated with tumor size (Figure 5c). Equal numbers of tumors were found in males and females, but consistent with the estrogenresponsiveness of the prolactin promoter used to drive transgene expression, the tumors were significantly larger in females, with some exhibiting intracranial extension and invasion of brain reminiscent of macroadenomas in humans (Figure 5d). Male animals developed multifocal microadenomas that could be seen on microscopic examination (Figure 5e); the tumors exhibited disruption of the normal acinar architecture of the pituitary, and prominent vascularity. The tumors were composed of lactotrophs with cytoplasmic immunoreactivity for prolactin (Figure 5f), and cytoplasmic FGFR4 reactivity consistent with ptdFGFR4 (Figure 5g). Other cell types of the pituitary were not affected. Age- and sex-matched nontransgenic littermates had no pituitary pathology. In contrast to animals expressing ptd-FGFR4, animals expressing the wild-type FGFR4 transgene had normal pituitary acinar architecture, with no evidence of hyperplasia or adenoma formation (Figure $5 \mathrm{~h}$ ), and a normal distribution of the various hormone-secreting cell types. The pituitaries exhibited a membrane-reactive pattern of FGFR4 immunopositivity (Figure $5 i$ ), with none of the cytoplasmic staining seen in ptd-FGFR4 transgenic mice; staining was restricted to lactotrophs that were normal in size, number, and distribution.

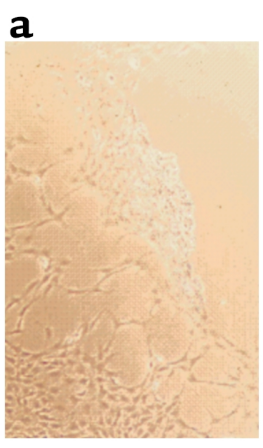

ptd-FGFR4

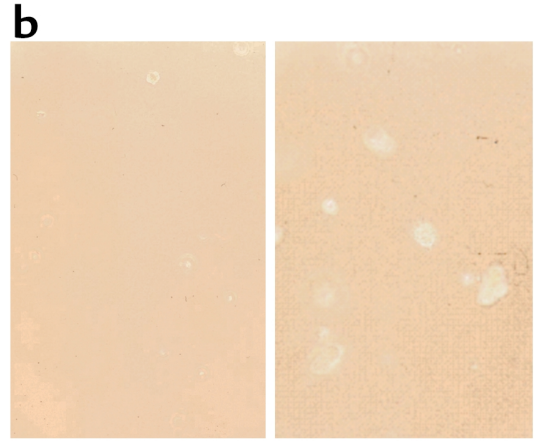

FGFR4 ptd-FGFR4

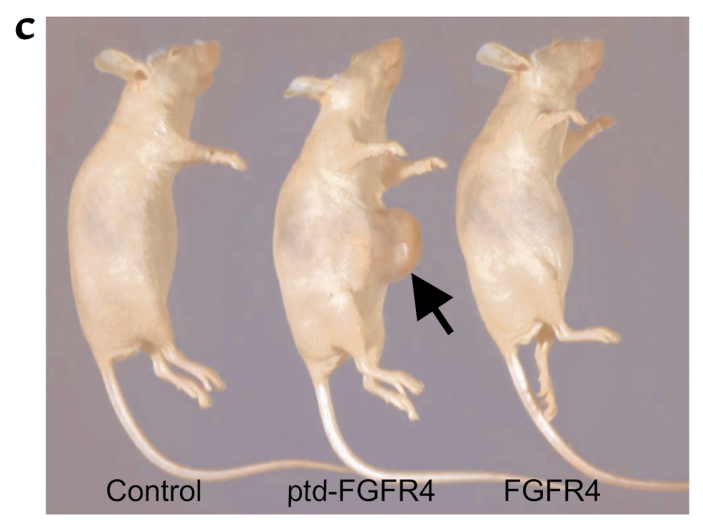

\section{Figure 4}

ptd-FGFR4 transforming ability in vitro and in vivo. (a) Anchorage independence in culture. NIH 3 T3 cells stably transfected with ptdFGFR4 lose contact inhibition. (b) Growth in soft agar. Cells stably transfected with FGFR4 rarely formed colonies (left), whereas those transfected with ptd-FGFR4 (right) formed numerous colonies greater than $50 \mu \mathrm{m}$ in diameter. (c) NIH $3 \mathrm{~T} 3$ cells stably transfected with ptdFGFR4 formed tumors within 2 weeks of injection. Cells transfected with empty vector or full-length FGFR4 did not form tumors. 

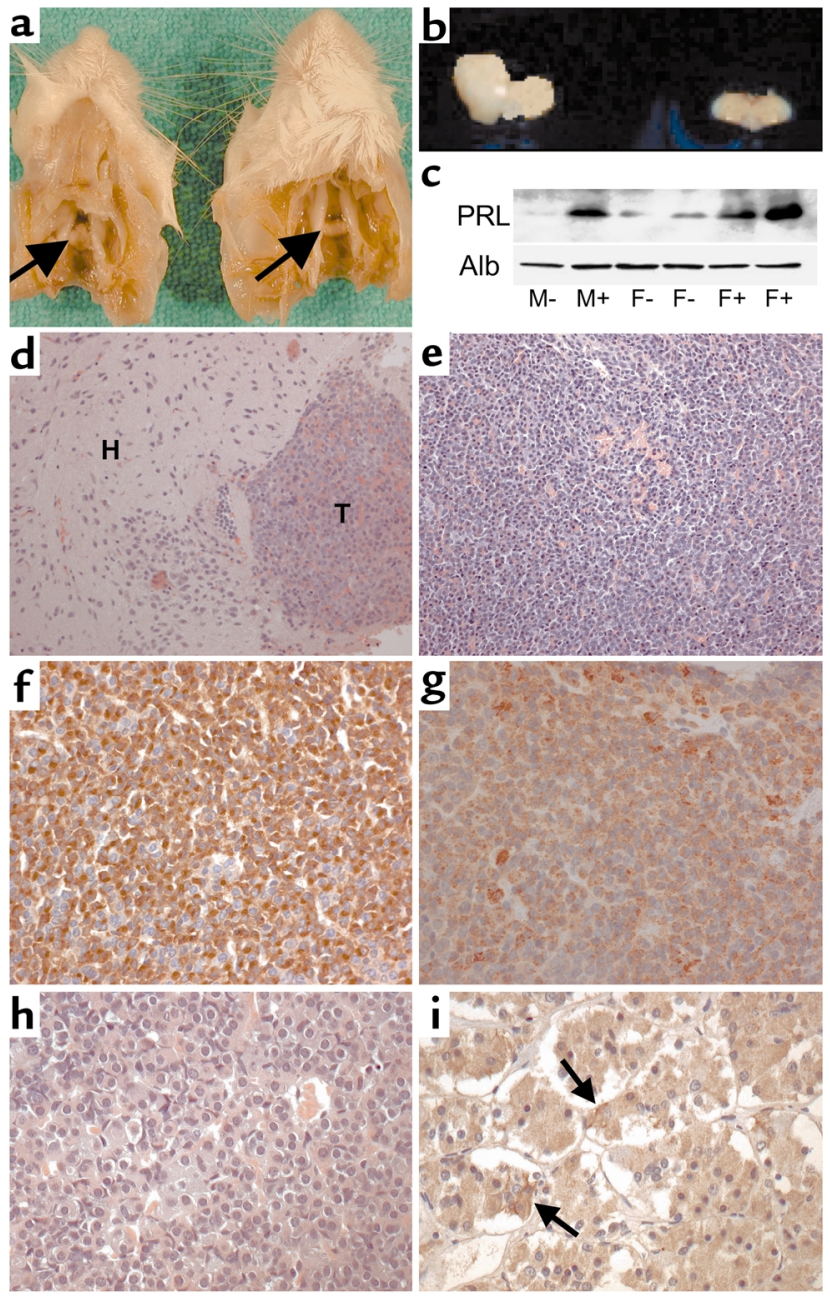

\section{Discussion}

The role of growth factors in pituitary cell proliferation and tumorigenesis has been the subject of much investigation (reviewed in ref. 2). A number of observations highlight the potential oncogenic contribution of the FGF system to pituitary tumorigenesis. At least 22 members of the FGF family have been described, with variable mitogenic, angiogenic, and hormone regulatory functions (14). One of these, FGF-2, was originally isolated from bovine pituitary (15) and is differentially expressed by pituitary tumor cells (16). FGF-2 is known to be angiogenic through stimulation of vascular EGF (26), suggesting a possible indirect mechanism for tumorigenesis. An N-terminally extended form of FGF-2 with a putative nuclear localization sequence has been reported in pituitary tumor cells (27). Human FGF-4 (bst) has been identified in transforming DNA derived from human pituitary tumors (28). Moreover, in one mouse model, estrogen-induced pituitary tumorigenesis was associated with enhanced expression of FGF-2 and of a pituitary tumor transforming gene (PTTG) (29).

FGF action is mediated by FGF receptors that exhibit a complex pattern of expression. Multiple forms of cellbound or secreted forms of FGFR1, -2, and -3 are gen-

\section{Figure 5}

Pituitary morphology of PRL-ptd-FGFR4 and PRL-FGFR4 transgenic mice. (a) The pituitary of an 11-month-old PRL-ptd-FGFR4 transgenic female mouse (left) is large, with nodular distortion; compare with nontransgenic female littermate (right). (b) The pituitary of an 11-monthold PRL-ptd-FGFR4 transgenic female mouse (left) is enlarged, with a tumor in the right lobe and a larger tumor replacing the left lobe. Normal pituitary of a nontransgenic female littermate (right). (c) Western blotting of mouse serum reveals higher levels of circulating PRL in transgenic mice (+) than in nontransgenic mice (-). The two left lanes are from males $(M)$; the four right lanes are from females $(F)$. Basal PRL is lower in the nontransgenic male than in the nontransgenic females. The degree of elevation in transgenic mice correlated with size of tumor. Albumen (Alb) control indicates protein loading (lower). (d) The pituitary tumor (T) of an 11-month-old PRL-ptd-FGFR4 transgenic female mouse invades the hypothalamus $(\mathrm{H})$. (e) The pituitary of an 11-month-old PRL-ptd-FGFR4 transgenic male mouse contains a tumor composed of small cells with chromophobic cytoplasm and prominent nuclei. There is loss of acinar architecture and increased vascularity. (f) Tumor cells contain PRL in PRL-ptd-FGFR4 transgenic mice. (g) Tumor cells in a PRL-ptd-FGFR4 transgenic mouse contain variable amounts of cytoplasmic FGFR4, similar to that seen in human tumors (Figure 2b). (h) The pituitary of a 12-month-old mouse transgenic for PRL-FGFR4 exhibits normal architecture and distribution of the various pituitary cell types. (i) Wild-type FGFR4 in a 12-month-old PRL-FGFR4 transgenic mouse has a membrane-staining pattern (arrows), and is found in scattered cells that correspond to lactotrophs.

erated from the same gene by alternative initiation, alternative splicing, exon shuffling, and/or variable polyadenylation (30). These isoforms differ in ligand binding and signaling potential, and their patterns of expression determine selective responsiveness of different tissues to different members of the FGF family. Initially, FGFR4 was considered unique in that the intact full-length receptor was believed to be the sole isoform. FGFR4 has 18 exons, in contrast to the 19 exons found in other FGFRs (19). The additional exon in FGFR1, -2, and -3 is located between exons 8 and 9 of FGFR4, and is used to generate alternatively spliced transcripts that encode isoforms of the third Ig-like domain. However, it is now recognized that the FGFR4 gene can also express isoforms. Human intestinal epithelial cells were recently shown to express an FGFR4 transcript in which exon 9 is displaced by intron 9 , leading to loss of the transmembrane domain (31). We identified expression of the N-terminal region of FGFR4 in normal pituitary, which does not express the kinase region of this gene, suggesting that FGFR4 may also derive a C-terminally truncated isoform (18). Subsequently, we showed that failure to splice intron 4 leads to an mRNA transcript encoding an in-frame premature stop codon, generating a soluble C-terminally truncated, dominant-negative FGFR4 isoform in human breast cancer cells (32). Alternative splicing of intron 17 in the mouse leads to a C-terminally truncated FGFR4 with a shorter intracellular tail (33); however, the functional significance of this FGFR4 splice variant is unknown.

FGFR4 is expressed mainly in adrenal, heart, lung, kidney, pancreas, muscle, and spleen, and was reported to be not expressed in brain (34). Our data indicate 
aberrant expression of a novel pituitary tumor-derived, $\mathrm{N}$-terminally truncated FGFR4 isoform that is not identified in the normal pituitary, providing a new ancillary diagnostic tool for these neoplasms. The mechanism underlying this phenomenon is not understood; we have excluded amplification or rearrangement as a causative mechanism. Further analysis will require characterization of the regulatory mechanisms underlying FGFR4 promoter activation in normal and abnormal circumstances.

Our current findings represent the first report of alternative transcription initiation in the genesis of an $\mathrm{N}$-terminally truncated FGFR4 isoform. This finding is consistent with the presence of a cryptic promoter within intron 4 of the FGFR4 sequence (19). The factors that are involved in the regulation of FGFR4 gene expression remain to be characterized, and the functional significance of this putative intronic promoter is not known.

The ptd-FGFR4 transcript that we have identified encodes a protein that includes the third Ig-like loop of the extracellular domain, the intracellular kinase region, and the $\mathrm{C}$-terminal portions of the protein, but not the signal peptide or two $\mathrm{N}$-terminal extracellular Ig-loops. The lack of a signal peptide is likely responsible for its localization in the cytoplasm. Since ptdFGFR4 contains a transmembrane domain, it is possible that it is trapped in a membrane-containing compartment of the cytoplasm, where by virtue of nonspecific oligomerization, it may become activated.

While our data indicate that the ptd-FGFR4 is constitutively phosphorylated, the tumorigenic potential of this receptor isoform may be enhanced by ligand, since growth in soft agar was slightly enhanced in the presence of FGF. This increase may be the result of interaction of ptd-FGFR4 with endogenous FGFRs in NIH $3 \mathrm{~T} 3$ cells. Nevertheless, expression of ptd-FGFR4 in NIH 3 T3 cells resulted in increased cell proliferation and anchorage independence in vitro, and caused tumorigenesis when injected in vivo. ptd-FGFR4 is not a potent transforming oncogene; this is consistent with the usually benign behavior of most pituitary tumors. Nevertheless, expression of ptd-FGFR4 in the pituitary of transgenic mice results in tumor formation, providing a rational basis for exploration of the potential utility of selective receptor kinase inhibitors in the treatment of pituitary adenomas.

In contrast to previous models of pituitary tumorigenesis in mice, the model we report here identifies a transforming oncogene that causes pituitary adenoma formation in the absence of prolonged and massive hyperplasia. There was no significant enlargement of the gland or expansion of pituitary acinar size in our animals prior to the formation of discrete adenomas. In previously reported models of pituitary tumorigenesis (4-9), there is a prolonged phase of massive hyperplasia of adenohypophysial cells associated with the primary stimulus. It has been argued that a "second hit" in the rapidly proliferating cells is required for neoplastic transformation (2). The model reported here lacks the phase of significant hyperplasia prior to the emergence of tumors, and therefore is much more analogous to the situation in human patients with pituitary adenomas.

In summary, we have identified, in human pituitary tumors, a novel $\mathrm{N}$-terminally truncated isoform of FGFR4, which we have named ptd-FGFR4. This truncated receptor lacks a signal peptide and is localized in the cytoplasm, where it exhibits ligand-independent tyrosine phosphorylation. Ectopic expression of ptdFGFR4 in NIH 3 T3 cells results in increased cell proliferation, anchorage independence, and colony formation in vitro, and causes tumorigenesis when injected in vivo. Pituitary expression of ptd-FGFR4, but not wild-type FGFR4, results in pituitary tumor formation in transgenic mice. This is the first report of an altered growth factor receptor isoform in primary human pituitary tumors that can be implicated in the neoplastic process in vivo. Moreover, this is the first time that a molecular alteration identified in sporadic human pituitary tumors has been shown to recapitulate in an animal model the morphologic and functional features of human pituitary disease.

\section{Acknowledgments}

The authors gratefully acknowledge Ali Abbass, Lily Ramyar, and Kelvin So for technical assistance. This work was supported by the Medical Research Council of Canada (grants MT-14404 to S. Ezzat, and MT14464 to S.L. Asa). ptd-FGFR4 sequence was deposited in GenBank under accession number AF359246.

1. Asa, S.L. 1998. Tumors of the pituitary gland. Atlas of tumor pathology, series 3, fascicle 22. Armed Forces Institute of Pathology. Washington, DC, USA. 214 pp.

2. Asa, S.L., and Ezzat, S. 1998. The cytogenesis and pathogenesis of pituitary adenomas. Endocr. Rev. 19:798-827.

3. Jacks, T., et al. 1992. Effects of an $R b$ mutation in the mouse. Nature. 359:295-300.

4. Kelly, M.A., et al. 1997. Pituitary lactotroph hyperplasia and chronic hyperprolactinemia in dopamine D2 receptor-deficient mice. Neuron. 19:103-113.

5. Asa, S.L., Kelly, M.A., Grandy, D.K., and Low, M.J. 1999. Pituitary lactotroph adenomas develop after prolonged lactotroph hyperplasia in dopamine D2 receptor-deficient mice. Endocrinology. 140:5348-5355.

6. Fero, M.L., et al. 1996. A syndrome of multiorgan hyperplasia with fea-

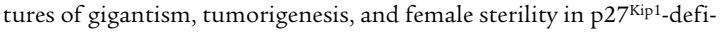
cient mice. Cell. 85:733-744.

7. Kiyokawa, H., et al. 1996. Enhanced growth of mice lacking the cyclin-

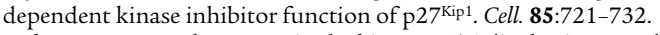

8. Nakayama, K., et al. 1996. Mice lacking $\mathrm{p}^{27^{\mathrm{Kip} 1}}$ display increased body size, multiple organ hyperplasia, retinal dysplasia, and pituitary tumors. Cell. 85:707-720.

9. Asa, S.L., et al. 1992. Pituitary adenomas in mice transgenic for growth hormone-releasing hormone. Endocrinology. 131:2083-2089.

10. Zhuang, Z., et al. 1997. Mutations of the MEN1 tumor suppressor gene in pituitary tumors. Cancer Res. 57:5446-5451.

11. Asa, S.L., Somers, K., and Ezzat, S. 1998. The MEN-1 gene is rarely downregulated in pituitary adenomas. J. Clin. Endocrinol. Metab. 83:3210-3212.

12. Vallar, L., Spada, A., and Giannattasio, G. 1987. Altered $G_{s}$ and adenylate cyclase activity in human GH-secreting pituitary adenomas. Nature. 330:566-568.

13. Ezzat, S., and Melmed, S. 1990. The role of growth factors in the pituitary. J. Endocrinol. Invest. 13:691-698.

14. Xie, M.-H., et al. 1999. FGF-19, a novel fibroblast growth factor with unique specificity for FGFR4. Cytokine. 11:729-735.

15. Gospodarowicz, D., Ferrara, N., Schweigerer, L., and Neufeld, G. 1987. Structural characterization and biological functions of fibroblast growth factor. Endocr. Rev. 8:95-114.

16. Ezzat, S., Smyth, H.S., Ramyar, L., and Asa, S.L. 1995. Heterogeneous in vivo and in vitro expression of basic fibroblast growth factor by human 
pituitary adenomas. J. Clin. Endocrinol. Metab. 80:878-884.

17. Givol, D., and Yayon, A. 1992. Complexity of FGF receptors: genetic basis for structural diversity and functional specificity. FASEB J. 6:3362-3369.

18. Abbass, S.A.A., Asa, S.L., and Ezzat, S. 1997. Altered expression of fibroblast growth factor receptors in human pituitary adenomas. J. Clin. Endocrinol. Metab. 82:1160-1166.

19. Kostrzewa, M., and Muller, U. 1998. Genomic structure and complete sequence of the human FGFR4 gene. Mamm. Genome. 9:131-135.

20. Tuominen, H., et al. 2001. Expression and glycosylation studies of human FGF receptor 4. Protein Expr. Purif. 21:275-285.

21. Johnston, C.L., Cox, H.C., Gomm, J.J., and Coombes, R.C. 1995. bFGF and aFGF induce membrane ruffling in breast cancer cells but not in normal breast epithelial cells: FGFR-4 involvement. Biochem. J. 306:609-616.

22. Johnston, C.L., Cox, H.C., Gomm, J.J., and Coombes, R.C. 1995. Fibroblast growth factor receptors (FGFRs) localize in different cellular compartments. J. Biol. Chem. 270:30643-30650.

23. Ornitz, D.M., et al. 1996. Receptor specificity of the fibroblast growth factor family. J. Biol. Chem. 271:15292-15297.

24. Olson, D.C., Deng, C., and Hanahan, D. 1999. Fibroblast growth factor receptor 4 , implicated in progression of islet cell carcinogenesis by its expression profile, does not contribute functionally. Cell Growth Differ. 9:557-564.

25. McAndrew, J., Paterson, A.J., Asa, S.L., McCarthy, K.J., and Kudlow, J.E. 1995 . Targeting of transforming growth factor- $\alpha$ expression to pituitary lactotrophs in transgenic mice results in selective lactotroph proliferation and adenomas. Endocrinology. 136:4479-4488.

26. Seghezzi, G., et al. 1998. Fibroblast growth factor-2 (FGF-2) induces vascular endothelial growth factor (VEGF) expression in the endothelial cells of forming capillaries: an autocrine mechanism contributing to angiogenesis. J. Cell Biol. 141:1659-1673.

27. Li, Y., et al. 1992. Identification and characterization of high molecular weight forms of basic fibroblast growth factor in human pituitary adenomas. J. Clin. Endocrinol. Metab. 75:1436-1441.

28. Gonsky, R., Herman, V., Melmed, S., and Fagin, J. 1991. Transforming DNA sequences present in human prolactin-secreting pituitary tumors. Mol. Endocrinol. 5:1687-1695.

29. Heaney, A.P., Horwitz, G.A., Wang, Z., Singson, R., and Melmed, S. 1999. Early involvement of estrogen-induced pituitary tumor transforming gene and fibroblast growth factor expression in prolactinoma pathogenesis. Nat. Med. 5:1317-1321.

30. Yan, G., et al. 1992. Expression and transformation of a variant of the heparin-binding fibroblast growth factor receptor $(\mathrm{flg})$ gene resulting from splicing of the exon at alternate $3^{\prime}$-acceptor site. Biochem. Biophys. Res. Commun. 183:423-430.

31. Takaishi, S., et al. 2000. Identification of a novel alternative splicing of human FGF receptor 4: soluble-form splice variant expressed in human gastrointestinal epithelial cells. Biochem. Biophys. Res. Commun. 267:658-662.

32. Ezzat, S., Zheng, L., Yu, S., and Asa, S.L. 2001. A soluble dominant negative fibroblast growth factor receptor 4 isoform in human MCF-7 breast cancer cells. Biochem. Biophys. Res. Commun. 287:60-65.

33. van Heumen, W.R.A., Claxton, C., and Pickles, J.O. 1999. Fibroblast growth factor receptor- 4 splice variants cause deletion of a critical tyrosine. Life. 48:73-78.

34. Hughes, S.E. 1997. Differential expression of the fibroblast growth factor receptor (FGFR) multigene family in normal human adult tissues. $J$. Histochem. Cytochem. 45:1005-1019. 